Bicocca-FT-07-6

CERN-PH-TH/2007-062

hep-ph/yymmnnn

\title{
RADIATIVE QUARKONIUM DECAYS AND THE NMSSM HigGS INTERPRETATION OF THE HYPERCP $\Sigma^{+} \rightarrow p \mu^{+} \mu^{-}$EVENTS
}

\author{
Michelangelo L. MANGANO ${ }^{(a)}$ and Paolo $\operatorname{NASON}^{(b)}$ \\ (a) CERN, PH-TH, Geneva, Switzerland \\ (b) INFN, Sezione di Milano Bicocca, Italy
}

\begin{abstract}
We study the potential of radiative decays of the $\Upsilon_{1 S}$ and of the $\phi$ mesons to search for a light pseudoscalar Higgs boson, proposed as a possible interpretation of $\Sigma^{+} \rightarrow p \mu^{+} \mu^{-}$events observed by the HyperCP collaboration at Fermilab. We conclude that the detection of this signal should certainly be possible with the current CLEO $\Upsilon_{1 S}$ data, and is within the reach of KLOE in at least part of the range of couplings suggested by the HyperCP findings.
\end{abstract}

\section{Introduction}

The HyperCP collaboration at Fermilab has recently reported [1] the observation of $3 \Sigma^{+} \rightarrow p \mu^{+} \mu^{-}$ decays, with the dimuon invariant mass $M_{\mu \mu}=214.3 \pm 0.5 \mathrm{MeV}$, consistent with the decay of a narrow neutral intermediate state $P^{0}$. Among the several candidate new particles put forward to explain this observation [2]- 7], the possibility [7] that $P^{0}$ is a light pseudoscalar Higgs $\left(a^{0}\right)$ in the next-to-minimal supersymmetric Standard Model (NMSSM) interestingly fits within theoretical frameworks recently explored in the attempt to loosen the very tight constraints set in the MSSM by the non-observation of the Higgs at LEP2.

Comprehensive studies of the implications of a light NMSSM Higgs for current and future measurements have been performed. Excellent examples are given, for example, in [ [8, 9] and in a comprehensive review of non-standard Higgs scenarios [10]. Here we limit ourselves to explore one important direct implication of the proposal made in [7]. Namely the possibility that $a^{0}$ be produced in quarkonium radiative decays $V \rightarrow \gamma a^{0}$ [11, where, in our analysis, $V=\Upsilon_{1 S}$ or $\phi(1020)$. The most recent and precise limit on $\Upsilon_{1 S} \rightarrow \gamma a^{0}$ was given by CLEO [12], where however $a^{0}$ is required to be stable and non-interacting. While several studies with visible Higgs final states have been performed since the early works of [13, we are not aware of limits valid in the case of prompt $a^{0} \rightarrow \mu^{+} \mu^{-}$decays in the range of branching ratios $\mathrm{B}\left(\Upsilon_{1 S} \rightarrow \gamma a^{0}\right)$ relevant to the model of [7. Likewise, we are not aware of experimental studies on the potential of $\phi$ decays to detect or constrain such scenarios. The excellent performance of the KLOE detector at DA $\Phi$ NE 
(see e.g. [14]), and the prospects for a continued operation at much higher luminosities1, make the consideration of $\phi$ decays extremely interesting.

Radiative quarkonium decays have been studied already in the context of other hypothetical interpretations of the HyperCP findings, namely the identification of $P^{0}$ with a sgoldstino [6]. In that case decay rates are typically beyond the present experimental sensitivities; in the case of a pseudoscalar Higgs, as we shall show, decay rates consistent with the HyperCP data are well within the reach of current experiments.

The virtue of our analysis is that it is only based on the assumptions on the coupling of $a^{0}$ to down-type quarks and to leptons, and it is independent of the details of the pattern of Higgs expectation values and mixings which need to be fixed to study other possible implications of this class of models [8, 9]. Furthermore, the quarkonium decay does not depend on the size of FCNC couplings of $a^{0}$, which enter in the analysis of the $\Sigma^{+} \rightarrow p \mu^{+} \mu^{-}$decay. Finally, the prediction for $\phi$ decays only depends on the $a^{0}$ coupling to the $s$ quark, which is what is probed in $\Sigma^{+} \rightarrow p \mu^{+} \mu^{-}$, and therefore evades the potential loophole of the $\Upsilon_{1 S}$ study, namely that in more complex Higgs sectors the coupling to the $b$ quark could be independent of the coupling to the $s$. Therefore, independently of the confirmation or invalidation of the hypothesis of [7], our note should be taken as an encouragement to revive the exploration of radiative quarkonium decays as a powerful tool to detect or constrain non-standard Higgs models, extending the considerations presented in ref. [8]. Other motivations to continue the experimental study of quarkonium radiative decays as a probe for new physics are presented in [15].

We start by briefly summarizing the key couplings of $a^{0}$ to the fermion sector.

$$
L_{a^{0} f \bar{f}}=-\left(g_{u} m_{u} \bar{u} \gamma_{5} u+g_{d} m_{d} \bar{d} \gamma_{5} d+g_{d} m_{\ell} \bar{\ell} \gamma_{5} \ell\right) \frac{i a^{0}}{v}
$$

where $v \sim 246 \mathrm{GeV}$, and the couplings $g_{u, d}$ depend on the details of the SUSY and EW symmetry breaking pattern. The analysis the HyperCP data in [7] suggests $g_{d}=\mathcal{O}(1)$, compatible with the constraint $\left|g_{d}\right| \lesssim 1.2$ from the muon $g-2$ [2]. A rough lower limit on $g_{d}$ can be obtained by requiring the Higgs lifetime to be short enough to guarantee the reconstruction of a common vertex for the three tracks relative to the muons and the proton in the HyperCP data. Using

$$
\Gamma\left(a^{0} \rightarrow \mu^{+} \mu^{-}\right)=\frac{g_{d}^{2}}{8 \pi} \frac{m_{\mu}^{2}}{v^{2}}\left[m_{a^{0}}^{2}-4 m_{\mu}^{2}\right]^{1 / 2}
$$

and using a Lorentz $\gamma$ factor of about 230 (the typical momentum of the dimuon pair in the HyperCP data is $50 \mathrm{GeV}[20]$ ), the average decay length of the $a^{0}$ in the laboratory frame turns out to be $\sim 0.02 \mathrm{~cm} / g_{d}^{2}$. Requiring this not to exceed a resolution in $z$ of the order of $60 \mathrm{~cm} \mathrm{[20],}$ would lead to $g_{d} \gtrsim 0.02$.

The above couplings induce quarkonium decays with a rate given by:

$$
\frac{B\left(V \rightarrow \gamma a^{0}\right)}{B\left(V \rightarrow e^{+} e^{-}\right)}=\frac{G_{F} m_{Q}^{2}}{\sqrt{2} \pi \alpha} g_{d}^{2}\left(1-\frac{m_{a^{0}}^{2}}{m_{V}^{2}}\right) F \sim 3.6 \times 10^{-4} \frac{m_{Q}^{2}}{\mathrm{GeV}^{2}} F g_{d}^{2}
$$

where $m_{Q}$ is the heavy quark mass and $F \sim 0.5$ includes the effect of QCD radiative corrections. For our numerical analysis, in the case of the $\Upsilon_{1 S}$ decays we'll use $m_{b}=M_{1 S} / 2$. In the case of

\footnotetext{
${ }^{1}$ See http://www.lnf .infn.it/lnfadmin/direzione/roadmap/roadmap.html
} 


\begin{tabular}{|l|c|}
\hline$\phi, 213.8 \mathrm{MeV}<M_{\mu \mu}<214.8 \mathrm{MeV}$ & $0.5 \mathrm{pb}$ \\
$S / \sqrt{B}$ for $2.5 \mathrm{fb}^{-1}$ & $1.8 g_{d}^{2}$ \\
\hline$\phi, 214.2 \mathrm{MeV}<M_{\mu \mu}<214.4 \mathrm{MeV}$ & $0.1 \mathrm{pb}$ \\
$S / \sqrt{B}$ for $2.5 \mathrm{fb}^{-1}$ & $3.9 g_{d}^{2}$ \\
\hline$\Upsilon_{1 S}, 2 m_{\mu}<M_{\mu \mu}<220 \mathrm{MeV}$ & $0.05 \mathrm{pb}$ \\
$S / \sqrt{B}$ for $1 \mathrm{fb}^{-1}$ & $150 g_{d}^{2}$ \\
\hline
\end{tabular}

Table 1: Cross section for the QED process $e^{+} e^{-} \rightarrow \mu^{+} \mu^{-} \gamma$, and significance of the $a^{0}$ signal.

the $\phi$, the small mass leads to a rather large uncertainty on the choice of the quark mass and of the size of QCD radiative corrections. As in the case of the $\Upsilon_{1 S}$, we shall adopt the convention $m_{s}=m_{\phi} / 2$, and discuss in appendix $\mathrm{B}$ the motivations and limitations of this choice. With these assumptions, and using $B\left(\Upsilon_{1 S} \rightarrow e^{+} e^{-}\right)=2.4 \times 10^{-2}$ and $B\left(\phi \rightarrow e^{+} e^{-}\right)=3 \times 10^{-4}$, we obtain:

$$
B\left(\Upsilon_{1 S} \rightarrow \gamma a^{0}\right)=1.9 \times 10^{-4} F g_{d}^{2} \quad, \quad B\left(\phi \rightarrow \gamma a^{0}\right)=2.8 \times 10^{-8} F g_{d}^{2}
$$

The rate of $\Upsilon_{1 S}$ produced at the $\mathrm{B}$ factories depends on the details of the beam energy resolution, which is typically larger than the intrinsic $\Upsilon_{1 S}$ width. Using the CLEO data discussed in [16] as a benchmark, we extract a production rate of $21 \times 10^{6} / 1.13 \mathrm{fb}^{-1} \sim 1.8 \times 10^{7} \mathrm{fb}$. A similar rate is expected for BaBar and Belle, due to comparable beam energy profiles. The DA $\Phi$ NE beams allow instead to operate at the peak of the $\phi$ resonance, with a rate given by:

$$
\sigma\left(e^{+} e^{-} \rightarrow \phi\right)=\frac{12 \pi}{m_{\phi}^{2}} B_{e^{+} e^{-}} A_{\mathrm{ISR}} \sim 2.8 \times 10^{9} \mathrm{fb}
$$

where $A_{\text {ISR }} \sim 2 / 3$ is the QED ISR correction factor. This leads to the following estimates for the number of $V \rightarrow \gamma a^{0} \rightarrow \gamma \mu^{+} \mu^{-}$decays per $\mathrm{fb}^{-1}$ (assuming $F=1 / 2$ ):

$$
\begin{aligned}
N\left(\Upsilon_{1 S} \rightarrow \gamma a^{0} \rightarrow \gamma \mu^{+} \mu^{-}\right) & =1700 g_{d}^{2} \\
N\left(\phi \rightarrow \gamma a^{0} \rightarrow \gamma \mu^{+} \mu^{-}\right) & =40 g_{d}^{2} .
\end{aligned}
$$

Considering that the current KLOE dataset consists of about $2.5 \mathrm{fb}^{-1}$, and that a factor of $>10$ increase is planned for the future, we conclude that both the B factories and DA $\Phi$ NE are potentially sensitive to the range of couplings required for the Higgs interpretation of the HyperCP events.

The actual observability of this signal depends however on the background rates. While the signature is rather sharp (a monochromatic photon, produced at large angle with energy close to $m_{V} / 2$ and recoiling against a low-mass dimuon pair), a potentially large background can arise from the continuum QED production of $e^{+} e^{-} \rightarrow \gamma \mu^{+} \mu^{-}$events. The actual size of the background will depend on the dimuon mass resolution, where the signal has a sharp peak with an intrinsic width below the $\mathrm{MeV}$. We point out that, for a muon pair produced near threshold, like in the case of the HyperCP signal, the invariant mass measurement should have an improved sensitivity. The invariant mass (as a function of the angular separation and the momentum of the muons) is in fact near a minimum, and therefore has a reduced sensitivity to the measured parameters and their errors, as discussed in an Appendix C. For our numerical study we assume dimuon mass resolutions equal to $\pm 5 \mathrm{MeV}$ for the $\Upsilon_{1 S}$, and $\pm 0.5 \mathrm{MeV}$ or $\pm 0.1 \mathrm{MeV}$ for the $\phi$. 
The resulting background rates are given in table 1, together with an estimate of the statistical significance of a possible signal. Here we required the photon to have energy larger than $1 \mathrm{GeV}$ $(100 \mathrm{MeV})$ for the $\Upsilon_{1 S}(\phi)$, and to be emitted at an angle with respect to the beam such that $|\cos \theta|<0.7$. Since the photon distribution in the signal is proportional to $1+\cos ^{2} \theta$, this cut has a signal efficiency of about 0.612 .

The separation of the signal from the background appears rather clear at the $\Upsilon_{1 S}$ with $1 \mathrm{fb}^{-1}$, even if the coupling is significantly smaller than its preferred $\mathcal{O}(1)$ value. The case for KLOE with its current statistics is at the borderline, even though there is a margin of uncertainty due to the large size of the QCD corrections. A mass resolution of $0.1 \mathrm{MeV}$ appears required to yield a significant signal.

Higher statistics, particularly at KLOE, would allow to strenghten the constraints on similar explorations of exotic BSM scenarios.

Acknowledgments We thank D. Asner, S. Bertolucci, F. Bossi, P. Dauncey, G. Isidori and H. Park for useful discussions and contributions to this study.

\section{A Background estimates}

The exact matrix element for the process $e^{-}\left(p_{1}\right) e^{+}\left(p_{2}\right) \rightarrow \gamma(k) \mu^{-}\left(q_{1}\right) \mu^{+}\left(q_{2}\right)$ can be easily calculated. We show here the result in the case of initial-state emission only, which is the relevant one for our signal:

$$
\begin{aligned}
\bar{\sum}_{p o l}\left|M\left(e^{+} e^{-} \rightarrow \gamma \mu^{+} \mu^{-}\right)\right|^{2}= & 4 \frac{e^{6}}{\left(p_{1} k\right)\left(p_{2} k\right)} \frac{1}{Q^{2}}\left[\left(p_{1} q_{1}\right)^{2}+\left(p_{2} q_{1}\right)^{2}+\left(p_{1} q_{2}\right)^{2}+\left(p_{2} q_{2}\right)^{2}+\right. \\
& \left.\frac{2 m_{\mu}^{2}}{Q^{2}}\left(\left(p_{1} Q\right)^{2}+\left(p_{2} Q\right)^{2}\right)\right]
\end{aligned}
$$

where $q_{1}+q_{2}=Q$. The final-state kinematics of the signal, two low-mass muons recoiling against the photon, allows us to approximate the cross section as:

$$
\frac{d \sigma\left(\gamma \mu^{+} \mu^{-}\right)}{d \cos \theta_{\gamma}}=\frac{d \sigma(\gamma \gamma)}{d \cos \theta_{\gamma}} \times \frac{d Q^{2}}{Q^{2}} \frac{\alpha}{2 \pi} P(z) d z
$$

where

$$
\frac{d \sigma(\gamma \gamma)}{d \cos \theta_{\gamma}}=2 e^{4} \frac{u^{2}+t^{2}}{u t} \frac{1}{32 \pi s}
$$

and

$$
P(z)=\left[z^{2}+(1-z)^{2}+2 \frac{m_{\mu}^{2}}{Q^{2}}\right]
$$

is the Altarelli-Parisi splitting function, for

$$
q_{1}^{\mu}=z Q^{\mu}, \quad \frac{1}{2}\left(1-\sqrt{1-\frac{4 m_{\mu}^{2}}{Q^{2}}}\right)<z<\frac{1}{2}\left(1+\sqrt{1-\frac{4 m_{\mu}^{2}}{Q^{2}}}\right) .
$$

\footnotetext{
${ }^{2}$ We verified that $S / \sqrt{B}$ is rather constant, and maximal, when the cut on $\cos \theta$ is varied in the region $0.6-0.8$.
} 
After integrating over $z$, over the dimuon mass in the range $2 m_{\mu}+\Delta_{1}<Q<2 m_{\mu}+\Delta_{2}$ (with $\Delta_{1,2}<<m_{\mu}$ ), and over the photon angular distribution in the range $-c<\cos \theta<c$, we obtain:

$$
\sigma\left(\gamma \mu^{+} \mu^{-}\right)=\frac{4 \pi \alpha^{2}}{s}\left(\log \frac{1+c}{1-c}-c\right) \frac{\alpha}{3 \pi} \frac{\Delta_{2}^{3 / 2}-\Delta_{1}^{3 / 2}}{m_{\mu}^{3 / 2}}
$$

While the results of table 1 were obtained using the exact formula, eq. (13) is an excellent approximation to the exact result, which can be used to easily explore the background size under cuts different from those used in table 1.

\section{B QCD corrections}

The QCD corrections to the $V \rightarrow P+\gamma$ decay of quarkonium are given by [19]

$$
\frac{\Gamma(V \rightarrow P+\gamma)}{\Gamma\left(V \rightarrow e^{+} e^{-}\right)}=\frac{G_{F} m_{q}^{2}}{\sqrt{2} \pi \alpha}\left(1-m_{P}^{2} / m_{V}^{2}\right)\left(1-\alpha_{s} C_{f} / \pi\left[a_{P}\left(\frac{E_{\gamma}}{E_{\gamma}^{\max }}\right)-\frac{1}{4}\right]\right)
$$

where we have assumed for simplicity $g_{d}=1$. The $-1 / 4$ term accounts for the QCD corrections to the $e^{+} e^{-}$decay rate. This result is obtained by renormalizing the quark mass in the on-shell scheme. Thus, the quark mass appearing in Wilczek formula is the pole mass; it can be given in terms of the $\overline{\mathrm{MS}}$ mass evaluated at the scale of the quark mass itself as [17]

$$
m_{q}=\bar{m}_{q}\left(\bar{m}_{q}\right)\left[1+C_{f} \alpha_{s} / \pi\right]
$$

so that eq. (14) becomes

$$
\frac{\Gamma(V \rightarrow P+\gamma)}{\Gamma\left(V \rightarrow e^{+} e^{-}\right)}=\frac{G_{F} \bar{m}_{q}^{2}\left(\bar{m}_{q}\right)}{\sqrt{2} \pi \alpha}\left(1-m_{P}^{2} / m_{V}^{2}\right)\left(1-\alpha_{s} C_{f} / \pi\left[a_{P}\left(\frac{E_{\gamma}}{E_{\gamma}^{\max }}\right)-2-\frac{1}{4}\right]\right),
$$

where $a_{P}$ is an increasing function of its argument, with a maximum value $a_{P}(1) \approx 6.62$. The strange $\overline{\mathrm{MS}}$ mass is determined to be around $0.1 \mathrm{GeV}$ at $2 \mathrm{GeV}$ [17]. Its value at a scale equal to the mass itself can be obtained using the renormalization group equation relation

$$
\frac{\bar{m}_{q}\left(\mu^{2}\right)}{\bar{m}_{q}\left(\mu_{0}^{2}\right)}=\left(\frac{\alpha_{s}\left(\mu^{2}\right)}{\alpha_{s}\left(\mu_{0}^{2}\right)}\right)^{4 / 9}
$$

and since $\alpha_{s}\left(\mu^{2}\right)$ diverges for $\mu<0.5 \mathrm{GeV}$, one would always determine $\bar{m}_{s}\left(\bar{m}_{s}\right)>0.5 \mathrm{GeV}$. Using 4-loop running for the strange mass [18, $\alpha_{s}\left(M_{Z}\right)=0.119$ and $\bar{m}_{s}(2 \mathrm{GeV})=0.1 \mathrm{GeV}$, we find $\bar{m}_{s}\left(\bar{m}_{s}\right)=0.6 \mathrm{GeV}$. The next question is what value of $\alpha_{s}$ one should use in eq. (16). The coefficient of $\alpha_{s}$ is $\approx 1.85$, and the scale of $\alpha_{s}$ should be taken around the annihilation energy. We find $\alpha_{s}(1 \mathrm{GeV}) \approx 0.5$, so that at this scale the radiative corrections has the same size of the Born term. Assuming that the correction exponentiates, we get $\exp (-0.5 \times 1.85) \approx 0.4$. Assuming instead a rational form $1 /(1+0.5 \times 1.85) \approx 0.5$. We thus conclude that setting the strange mass to half of the $\phi$ mass, and using a correction factor of order of a half yields a reasonable estimate. We should not forget, however, that uncertainties are very large, so that a rate a few times larger or smaller than our estimate is not unconceivable. 


\section{C $\mu^{+} \mu^{-}$kinematics near threshold}

The invariant mass of the two muons, as a function of their momenta $p_{1}, p_{2}$ and thair angular separation $\theta$ is

$$
\begin{aligned}
M_{\mu \bar{\mu}}^{2} & =4 m_{\mu}^{2}+2 p_{1} p_{2}(1-\cos \theta)+2\left[E_{1} E_{2}-p_{1} p_{2}-m_{\mu}^{2}\right] \\
& =4 m_{\mu}^{2}+2 p_{1} p_{2}(1-\cos \theta)+2 m_{\mu}^{2} \frac{\left(p_{1}-p_{2}\right)^{2}}{E_{1} E_{2}+p_{1} p_{2}+m_{\mu}^{2}} .
\end{aligned}
$$

Near threshold, the second and third terms in eq. (18) become small, so that in this limit

$$
M_{\mu \bar{\mu}} \approx 2 m_{\mu}+\frac{p^{2}}{4 m_{\mu}} \theta^{2}+\frac{m_{\mu}}{4} \frac{\Delta p^{2}}{E^{2}},
$$

where $p$ is the common value of $p_{1}$ and $p_{2}, E$ is their energy, and $\Delta p=p_{1}-p_{2}$ is their small momentum difference. Experimental errors $\delta \theta$ and $\delta \Delta p$ on the measurement of the angle and the momentum affect the invariant mass measurement by

$$
\delta M_{\mu \bar{\mu}} \approx \frac{p^{2}}{2 m_{\mu}} \theta \delta \theta+\frac{m_{\mu}}{2} \frac{\Delta p}{E^{2}} \delta \Delta p .
$$

Thus the error is reduced by the small factors $\theta$ and $\Delta p$.

\section{References}

[1] H. Park et al. [HyperCP Collaboration], Phys. Rev. Lett. 94 (2005) 021801 arXiv:hep-ex/0501014.

[2] X. G. He, J. Tandean and G. Valencia, Phys. Lett. B 631 (2005) 100 arXiv:hep-ph/0509041].

[3] N. G. Deshpande, G. Eilam and J. Jiang, Phys. Lett. B 632 (2006) 212 arXiv:hep-ph/0509081].

[4] C. Q. Geng and Y. K. Hsiao, Phys. Lett. B 632 (2006) 215 arXiv:hep-ph/0509175.

[5] D. S. Gorbunov and V. A. Rubakov, Phys. Rev. D 73 (2006) 035002 arXiv:hep-ph/0509147].

[6] S. V. Demidov and D. S. Gorbunov, JETP Lett. 84 (2007) 479 arXiv:hep-ph/0610066.

[7] X. G. He, J. Tandean and G. Valencia, Phys. Rev. Lett. 98, 081802 (2007) |arXiv:hep-ph/0610362.

X. G. He, J. Tandean and G. Valencia, Phys. Rev. D 74, 115015 (2006) arXiv:hep-ph/0610274].

[8] R. Dermisek, J. F. Gunion and B. McElrath, arXiv:hep-ph/0612031.

[9] G. Hiller, Phys. Rev. D 70 (2004) 034018 arXiv:hep-ph/0404220].

[10] E. Accomando et al., arXiv:hep-ph/0608079.

[11] F. Wilczek, Phys. Rev. Lett. 39 (1977) 1304.

[12] R. Balest et al. [CLEO Collaboration], Phys. Rev. D 51 (1995) 2053.

[13] P. Franzini et al., Phys. Rev. D 35 (1987) 2883.

[14] F. Ambrosino et al. [By KLOE Collaboration], arXiv:hep-ex/0701008.

[15] M. A. Sanchis-Lozano, arXiv:hep-ph/0610046.

[16] D. Besson et al. [CLEO Collaboration], arXiv:hep-ex/0512003.

[17] A.V. Manohar and C.T. Sachrajda, "Quark Masses", in the "The Review of Particle Physics", W.M. Yao et al., J. Phys. G 33, 1 (2006).

[18] J. A. M. Vermaseren, S. A. Larin and T. van Ritbergen, Phys. Lett. B 405 (1997) 327 arXiv:hep-ph/9703284.

[19] P. Nason, Phys. Lett. B 175 (1986) 223.

[20] H. Park, private communication. 